vehicle during its initial encounter with the comet while at a long distance from the Sun.

The mission has been redefined without the sample-return element, reducing its costs to an estimated 600 million units of account (US $\$ 720$ million). But its technical feasibility, scientific value and reduced cost are now such that European scientists are confident the mission can be accomplished without the aid of NASA.

As a result, ESA's space science advisory committee decided at a meeting last month to recommend an earlier than anticipated launch date of 2003 , with the initial comet rendezvous scheduled for 2008 .

The main space vehicle will travel in parallel with the comet until the two reach the comet's perihelion in 2011. Proposals are now being discussed with scientific groups and funding agencies in various countries - including the United States, Russia and Japan, as well as ESA member states --. to build instruments for a separate vehicle that would be dropped on to the comet's surface to make physical and chemical measurements.

Planetary scientists are pleased with the priority now being given to the Rosetta mission. "I am very excited by the prospects", says Eberhardt Grün of the Max Planck Institute for Nuclear Physics in Heidelberg, who has been working on plans for the mission for almost ten years.

US scientists, frustrated by NASA's decision last year to drop funding for its own proposed comet rendezvous mission, CRAF, are also looking forward to the opportunity they are being offered to participate in a cometary mission, even one in which Europe will now be setting the timetable.

"For example, an instrument developed for the CRAF mission designed to send back images of the surface could now be used on Rosetta", says Thomas Ahrens of the Seismological Laboratory at the California Institute of Technology, one of the main authors, with Grün, of a 1987 report outlining the scientific goals of the mission.

The main disappointment will come from European astronomers, however, who had been hoping that the third cornerstone launch slot would be given to the Far Infrared Submillimetre Wave Telescope, FIRST (the first two slots are for the $\mathrm{SOHO} /$ Cluster space vehicles, and the XMM telescope).

After a presentation of both Rosetta and FIRST, ESA's advisory committee decided at last month's meeting to recommend that the Rosetta mission be launched first, in which case the telescope will not be launched until at least 2006. One factor said to have influenced the committee's decision is that there are a number of astronomy missions already waiting to be launched.

Both decisions are expected to be endorsed by ESA's science programme committee when it meets in Paris on 4 November to discuss the advisory committee's recommendations.

David Dickson

\title{
Italy cuts research funds but adds university jobs
}

Rome. The Italian government is to create thousands of new university teaching and research posts to meet rising student demand, even though it is planning to cut the national research budget by 3 per cent and is recommending even larger cuts in its direct funding of university research.

The cuts are being imposed as part of a package of stringent measures intended to deal with the country's economic crisis. Nevertheless, Umberto Colombo, the research minister, has managed to implement a decision made as part of a previously agreed three year plan for 1991-93 (but blocked by bureaucracy) to increase the number of permanent university staff

As a result, funding for 1,250 full and associate professors, and for 2,000 research staff, will be allocated to universities by the end of the year. Discussions are taking place between the Ministry of Universities and Research, the National Research Council and universities to decide the precise number of new posts to be given to individual institutions.

In a separate move, universities and research institutes were told two weeks ago that they are to be exempted from the government's freeze on all recruitment to civil servant posts, allowing them to fill existing vacancies.

Both measures have been prompted by the fact that the number of Italian university students has tripled in the last 25 years, to a total of 1.5 million. But over the same period, there has been no accompanying increase in teaching staff; the number of professors, for example, has remained essentially unaltered at 45,000 .

Despite staffing improvements, the government is recommending that the funds universities devote next year to research should be reduced by 15 per cent on 1993 . Colombo has long criticized universities for using these funds in an unfocused way without adequate targeting (see Nature 364, 473; 1993).

But Colombo also points out that he is now giving universities more control over

\begin{tabular}{|lrr|}
\hline Research institutes facing funding cuts \\
& $\underline{\mathbf{1 9 9 3}}$ & $\mathbf{1 9 9 4}$ \\
National Research Council & 1110 & 1050 \\
Italian Space Agency & 850 & 800 \\
Nuclear and Alternative Energy Agency & 600 & 550 \\
National Institute for Nuclear Physics & 440 & 400 \\
\hline
\end{tabular}

All figures in billions of tire.

the way they allocate their budgets, which will be provided by the government as a lump sum. This means that they can choose to increase the government's suggested budget for basic research with funds saved from other administrative areas.

The overall research budget is likely to be at least 3 per cent lower than last year. The first round of cabinet debate on the budget has left the research ministry with much less than it had hoped for. As a result, says Colombo, "small sacrifices will have to be made in nearly all areas". But he says that, in comparison with other ministries, which have had to accept cuts averaging at about 6 per cent, research has not done too badly. The budget should receive final approval from the parliament by the end of the year.

Colombo also points out that he has managed to secure an increase in funds for capital investments in research facilities. These will allow for the completion on the national Synchrotron Radiation Facility in Trieste, and building of the national telescope Galileo.

Luciano Maiani, head of the National Institute for Nuclear Physics (INFN), says that the cuts hurt, but acknowledges that they have been made necessary by the economic situation. But he says that INFN will be able to absorb the cuts without a major impact on its research activities.

The INFN, which runs four institutes and 12 university research units with at least 800 scientists, is now in the first year of a fiveyear plan introduced by Maiani. His budget for next year has now been cut back by 10 per cent

To meet the new budget targets, Maiani has delayed two long-term projects to construct powerful heavy-ion accelerators. But time has also been bought by continuing delays to the proposed Large Hadron Collider (LHC) at the European particle physics laboratory (CERN) in Geneva, now expected to be ready by 2001 if it is approved at CERN council this December.

As a result, INFN has been able to put back the construction of LHC detectors for which it is responsible. And Maiani says that he has been reassured by - even though he is not counting on - promises from the ministry that the 10 per cent cut will be made up over the following two years.

Alison Abbott 